www.nature.com/pj

\title{
Preparation and characterization of organic-inorganic microcomposite cylindrical GRIN lens
}

\author{
Eisuke Nihei, Junichi Oomoto, Soichiro Kimura and Koichi Asakura
}

\begin{abstract}
Although many studies on graded index (GRIN) lenses have been reported over the past several years, it is extremely difficult to find reports that have used polymer GRIN lenses in an atmosphere involving a large temperature change, which is mainly because of their lack of thermal stability. In this study, we propose a new method of fabricating organic-inorganic microcomposite cylindrical GRIN lenses with high thermal stability. This method is based on the spontaneous frontal polymerization (SFP) technique and diffusion of two organic materials with different refractive indices into an inorganic wet-gel. The refractive index profile of the GRIN lens is made by this diffusive process. We are able to determine the relationship between the refractive index profile and the diffusion time using our original refractive index profile simulation, based on the diffusion equation. The obtained microcomposite cylindrical GRIN lenses are not only thermally stable but also have a controlled refractive index profile, due to their inorganic matrix. The lenses are also highly transparent-an essential factor. The SFP technique also enabled high reproducibility of microcomposite cylindrical GRIN lenses with smooth periphery in this study, indicating the high potential of this process for practical application.
\end{abstract}

Polymer Journal (2010) 42, 941-946; doi:10.1038/pj.2010.105; published online 27 October 2010

Keywords: diffusion equation; organic-inorganic microcomposite; refractive index profile; spontaneous frontal polymerization

\section{INTRODUCTION}

Lenses with a refractive index gradient are called graded index (GRIN) lenses. GRIN lenses have excellent optical characteristics compared with spherical/aspherical lenses that consist of a material with a uniform refractive index. Hence, many researchers are motivated to expand the understanding of this particular type of lens. ${ }^{1,2,3}$

Generally, the refractive index profile of the cylindrical GRIN lens is expressed by

$$
n(r)=n_{0}\left(1-\frac{1}{2} A r^{g}\right)
$$

where $r$ is the distance from the cylindrical axis, $A$ is the positive constant, and $n(r)$ and $n_{0}$ are the indices at the radius $r$ and on the axis, respectively. Variable $g$ is a refractive index profile coefficient (index exponent), which characterizes the refractive index profile. The optimum value of $g$ for a GRIN lens is about 2.,5

The easy handling of organic material has also helped broaden this field of study. However, the low thermal stability of organic cylindrical GRIN lenses has always been a major issue for practical application. Cylindrical GRIN lenses made of inorganic materials with high thermal stability could be made only with a diameter of several millimeters at most, which is also undesirable for practical application factor. ${ }^{6}$ In this study, we present a new method, the spontaneous frontal polymerization (SFP) technique, for the fabrication of organic-inorganic microcomposite cylindrical GRIN lenses with high thermal stability and a large diameter $(35 \mathrm{~mm})$. In this SFP technique, the degree of polymerization advances after a certain period of time from the initiation of polymerization, which is accompanied by an increase in viscosity of the polymerization solution. The heat of polymerization, therefore, cannot be easily discharged. As a result, the autoacceleration polymerization reaction occurs near the central region of the container. ${ }^{7}$ This phenomenon is called the thermal storage effect, and the difference in refractive index of the high conversion area and low conversion area appears as a border plane. This plane is observed as a front that progresses from the center of the container toward the peripheral region as polymerization proceeds. (This type of polymerization is referred to as 'frontal polymerization.') We also present a refractive index distribution control method using diffusion. In addition, we clarify the optical characteristics of the microcomposite cylindrical GRIN lens obtained by the SFP technique.

\section{EXPERIMENTAL PROCEDURE}

Materials

Tetramethyl orthosilicate (Tokyo Chemical Industry, Tokyo, Japan) was used as an inorganic wet-gel without further purification. Anhydrous ethanol (EtOH; Wako Pure Chemical Industries, Ltd., Osaka, Japan) was used as the solvent, and a small amount of ammonia solution (aqueous $\mathrm{NH}_{4} \mathrm{OH}$; Wako Pure

Center for Science and Technology for Designing Functions, School of Integrated Design Engineering, Graduate School of Science and Technology, Keio University, Yokohama, Japan

Correspondence: Dr E Nihei, Center for Science and Technology for Designing Functions, School of Integrated Design Engineering, Graduate School of Science and Technology, Keio University, 3-14-1 Hiyoshi, Kohoku-ku, Yokohama 223-8522, Japan.

E-mail: eisuke@appi.keio.ac.jp

Received 7 July 2010; revised 18 September 2010; accepted 20 September 2010; published online 27 October 2010 
Table 1 Materials used for organic-inorganic microcomposite graded index lens and their physical properties

\begin{tabular}{llcc}
\hline Material & Function & $\begin{array}{c}\text { Refractive } \\
\text { index } \\
\text { (polymer) }\end{array}$ & $\begin{array}{c}\text { Density }\left(\mathrm{gcm}^{-3} \text { ) }\right. \\
\text { (polymer) }\end{array}$ \\
\hline $\begin{array}{l}\text { Tetra-methyl ortho-silicate } \\
\text { Methyl methacrylate }\end{array}$ & Monomer & 1.460 & - \\
$\begin{array}{l}\text { Styrene } \\
\text { 2,2,3,3,-Tetrafluoropropyl }\end{array}$ & Monomer & 1.492 & 1.23 \\
methacrylate & Monomer & 1.592 & 1.05 \\
Ethylene glycol dimethacrylate & Monomer & 1.424 & 1.54 \\
Benzoyl peroxide & Crosslinker & 1.506 & 1.24 \\
\hline
\end{tabular}

Chemical Industries, Ltd.) was used as a catalyst without further purification. Size 4A molecular sieves (Junsei Chemical Co., Ltd., Tokyo, Japan) were used for the absorption of EtOH. Methyl methacrylate (MMA; Mitsubishi Rayon Co., Ltd., Tokyo, Japan), styrene (St; Junsei Chemical Co., Ltd., Tokyo, Japan) and 2,2,3,3-tetrafluoropropyl methacrylate (4FMA; Mitsubishi Rayon Co., Ltd.) were used as monomers. Ethylene glycol dimethacrylate (Wako Pure Chemical Industries, Ltd.) was used as a crosslinking agent. Monomers and the crosslinking agent were both purified by distillation at reduced pressure. Benzoyl peroxide (Nacalai Tesque, Inc., Kyoto, Japan) was used as an initiator without further purification. The function and properties of each material are summarized in Table 1.

Fabrication of organic-inorganic microcomposite cylindrical GRIN lenses

First, a wet-gel was prepared by dissolving tetramethyl orthosilicate and $\mathrm{H}_{2} \mathrm{O}$ in $\mathrm{EtOH}$, with a small amount of aqueous $\mathrm{NH}_{4} \mathrm{OH}$ as catalyst, in a glass tube with a $15-\mathrm{mm}$ diameter. The molar ratio composition of this solution was tetramethyl orthosilicate/EtOH/ $\mathrm{H}_{2} \mathrm{O}=1: 5: 4$. The reactive solution was agitated for $48 \mathrm{~h}$ at room temperature, gradually forming a gel. The obtained silica wet-gel was then washed by EtOH to remove residual tetramethyl orthosilicate and catalyst, and soaked in M1 monomer twice (each for $48 \mathrm{~h}$ at room temperature) to replace EtOH with M1 monomer. Here, MMA was used as the M1 monomer, which is composed of $10 \mathrm{wt} \%$ crosslinking agent and $1.0 \mathrm{wt} \%$ initiator. The silica wet-gel was completely saturated during this soaking process. Molecular sieves were also used as an absorption agent for EtOH. The gas chromatography method confirmed that the amount of ethanol dissolved in the surrounding material was $0.8 \mathrm{wt} \%$ or less by the second soaking.

After the wet-gel was thoroughly soaked in M1 monomer, it was polymerized in air or in water to fabricate a microcomposite sample for the evaluation of its transparency. Unfortunately, the obtained silica wet-gel often cracked when polymerized in air or in water, making the sample polymers inadequate for experimental use. Therefore, it was necessary to polymerize the gel in M1 monomer to avoid fabrication of such defective samples. However, it is extremely difficult to detach the polymerized gel from the polymerizing M1 monomer surrounding it. To overcome this difficulty, the SFP technique was used to polymerize only the gel contained within the M1 monomer. ${ }^{7}$

During the SFP technique, the surrounding monomer cannot polymerize, therefore, the polymerized gel was easily extracted. In this study, the wet-gel was polymerized by the SFP technique in a constant-temperature bath at $60^{\circ} \mathrm{C}$, and the percentage of the monomer that diffused into an inorganic wet-gel was about $85 \%$ of the obtained sample. The obtained sample was then heat treated for $24 \mathrm{~h}$ at $70^{\circ} \mathrm{C}$ and for another $24 \mathrm{~h}$ at $90^{\circ} \mathrm{C}$. The sample was of a cylindrical form with a $14-\mathrm{mm}$ diameter and a $10-\mathrm{mm}$ thickness. Its transmittance was measured using a spectrophotometer (Hitachi U-1000, Hitachi, Ltd., Tokyo, Japan), and the temperature dependence of its refractive index (thermo-optical coefficient (TOC)) was measured by an Abbe refractometer (NAR-1T, ATAGO Co., Ltd., Tokyo, Japan).

Another microcomposite GRIN lens sample was fabricated from the wet-gel thoroughly soaked in M1 monomer. This sample was fabricated by diffusing a different monomer (M2) from the peripheral region of the gel. Here, 4FMA was used as the M2 monomer. Two diffusion times were set for this experiment ( 0 and $60 \mathrm{~min}$ ). During this process, a mutual diffusion occurred between the two monomers, thus forming a refractive index gradient within the gel.

This sample was also heat treated for $24 \mathrm{~h}$ at $70^{\circ} \mathrm{C}$ and another $24 \mathrm{~h}$ at $90{ }^{\circ} \mathrm{C}$ after polymerization and then cut into a disk shape of about $1 \mathrm{~mm}$ in thickness. The refractive index profile of the microcomposite sample made by M1 and M2 monomers was measured by an interference microscope (Carl Zeiss Jena Interphako, Carl Zeiss AG, Oberkochen, Germany). The value of the $g$ was determined by fitting the obtained profile data using the Gnuplot program.

\section{RESULTS AND DISCUSSION \\ SFP}

To observe the formation of the front in the center region, a wet-gel consisting only of MMA was polymerized at $60^{\circ} \mathrm{C}$. Figure 1 shows a photograph taken from the side of the glass vessel placed in a constant-temperature bath. The elapsed time from the initiation of the polymerization is indicated at the lower right of each figure. At $20 \mathrm{~min}$ after the initiation of polymerization, the wet-gel became translucent (refer to Figure 1, at $1354 \mathrm{~s}$ ). After another $4 \mathrm{~min}$, the front clearly forms at the center of the wet-gel (refer to Figure 1, at $1440 \mathrm{~s}$ ). From these results, it could be assumed that polymerization begins $20 \mathrm{~min}$ after initiation. As mentioned before, the SFP technique allows the polymerization reaction to begin from the center of the vessel, without complex procedures. Hence, it is possible to polymerize only the microcomposite material and not the surrounding monomer.

\section{Transparency of microcomposite materials}

As shown in Figure 2, the obtained microcomposite GRIN lens has no haze, which indicates its capability for optical applications. Figure 3 shows the spectral transmittance of the microcomposite material and poly(methyl methacrylate) (PMMA). The microcomposite material is visibly transparent as is PMMA. The transparency does not exceed $90 \%$ because of the reflection effect. Surface reflectance is $\sim 4 \%$ on one surface and $10 \%$ or less on both surfaces when the refractive index of the sample is 1.5 . Therefore, it is assumed that there was hardly any absorption or light scattering. High transmittance also shows that the difference in microheterogeneous structure between the organic phase and inorganic phase is small. Furthermore, the value of the refractive index of PMMA $(n=1.49)$ and $\mathrm{SiO}_{2}(n=1.46)$ is close, which limits the influence on light scattering. Notably, microcomposite materials have a lower transparency than PMMA in the short wavelength region (300-500 nm), which is most likely the effect of Rayleigh scattering. In this case, Rayleigh scattering is inversely proportional to wavelength by the fourth power, caused by the generation of an inhomogeneous region smaller than the wavelength order and in the matrix of the inorganic material.

\section{The thermal stability of the microcomposite lens}

The TOC was derived from measurements by a temperature-controlled Abbe refractometer. The plot in Figure 4 shows the relationship between the refractive index and temperature of PMMA. The slope of this plot is the TOC. The obtained TOC of PMMA was $1.1 \times 10^{-4} \mathrm{~K}^{-1}$, which is similar to previously reported data $\left(1.1-1.2 \times 10^{-4} \mathrm{~K}^{-1}\right){ }^{8}$

Figure 5 shows the dependency of refractive index variation on the temperature for the microcomposite material $\left(\mathrm{SiO}_{2}+\mathrm{PMMA}+\right.$ poly(ethylene glycol dimethacrylate) (PEGDMA)) and a typical organic material (PMMA+PEGDMA). These materials were fabricated under similar conditions to those of the lens. The TOC of the microcomposite material was $6.9 \times 10^{-5} \mathrm{~K}^{-1}$ and that of organic material was $8.0 \times 10^{-5} \mathrm{~K}^{-1}$. Here, it can be clearly seen that the 


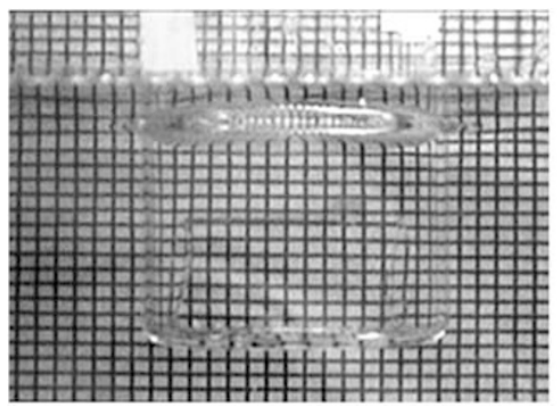

750 sec.

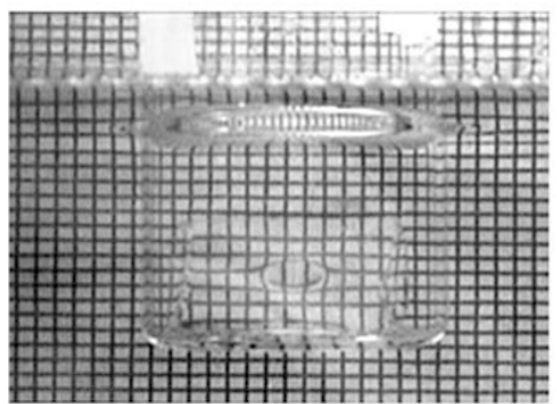

1440 sec.

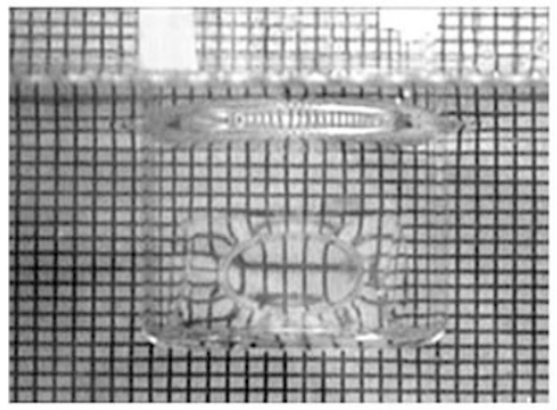

1472sec.

Figure 1 Photograph of the front formation in the SFP technique.

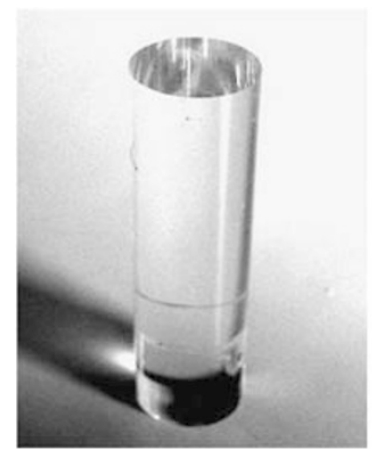

Illel luiny al ive organic phase occu mainer small to atter of ( $\left.\mathrm{SA}^{-} \mathrm{s}\right)$ wa mpl PMM 13 A-: atter of cure was c intradi. postula1 ze is on the order of 1

- the raciulte wrac no

Figure 2 The appearance of fabricated organic-inorganic microcomposite graded index (GRIN) lens (left) and the image observed through the obtained GRIN lens (right).

organic material has a TOC that is about $14 \%$ smaller than the microcomposite material. For organic polymers, the TOC exclusively depends on its volume expansion coefficient. ${ }^{8}$ From these results, it was assumed that the $\mathrm{SiO}_{2}$ network inhibited the thermal expansion of

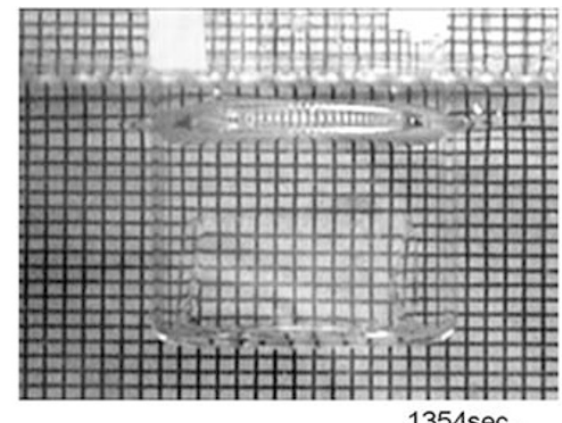

$1354 \mathrm{sec}$.

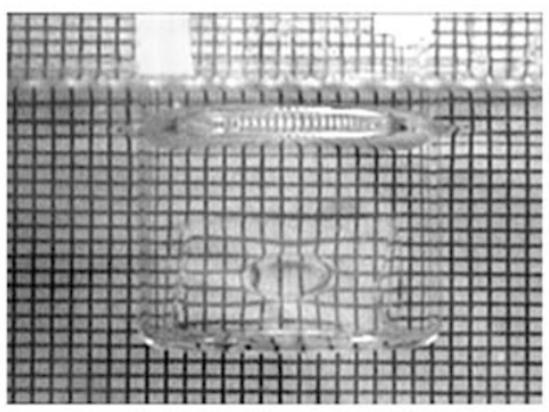

1445 sec.

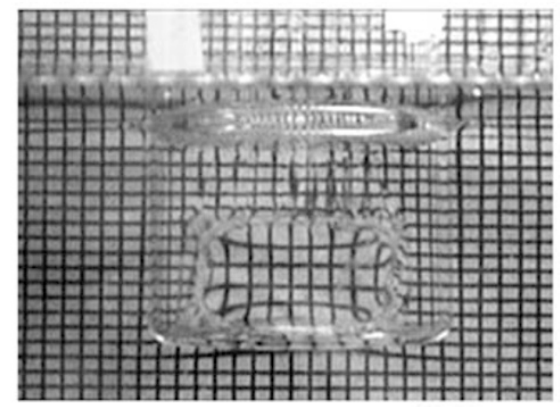

1538 sec.

organic polymers. The composition ratio of $\mathrm{SiO}_{2}$ in the microcomposite material was $15 \%(\mathrm{w} / \mathrm{w})$, which almost corresponds to the decrease in TOC. These results also indicate that almost all of the monomer components polymerized. Furthermore, the weight change of the obtained sample was $<1 \mathrm{wt} \%$ and deformation was not observed after $48 \mathrm{~h}$ of heat treatment at $120^{\circ} \mathrm{C}$. In other words, the increase in the ratio of $\mathrm{SiO}_{2}$ will further decrease the TOC and improve thermal stability.

\section{Control of refractive index profiles}

In order to use the GRIN lens as an optical component, the refractive index profile of the GRIN optical element needs to be controllable. Contrary to the conventional method, our proposed method allows easy control of formation of the refractive index profile. In this study, the refractive index profile was easily manipulated by controlling the monomer diffusion because the refractive index profile was achieved by changing the composition distribution of monomers. Thus, simulations based on the diffusion equation were first executed to provide further insight into the possibility to control the refractive index distribution. 


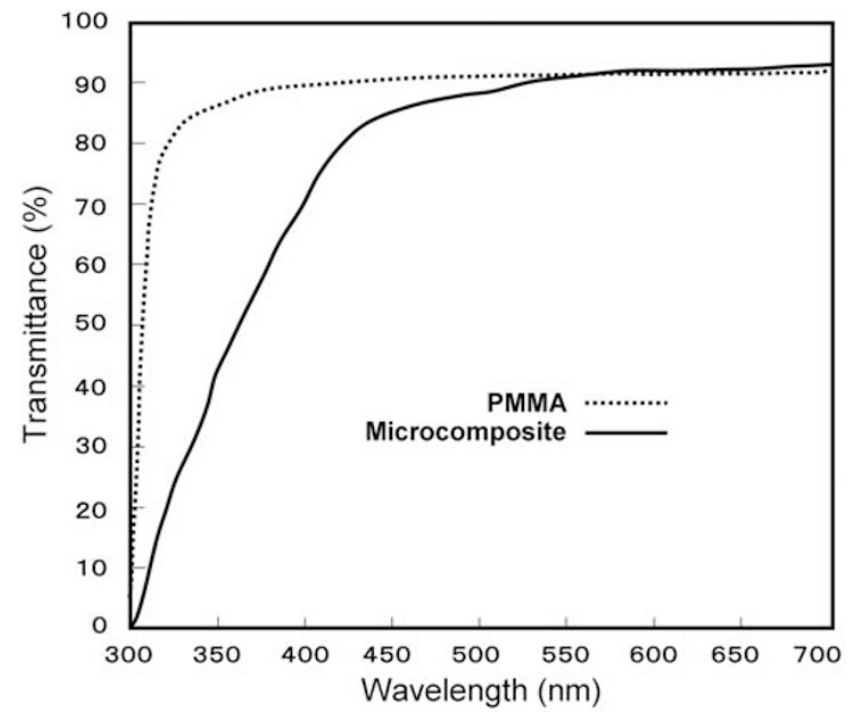

Figure 3 Comparison of the spectral transmittance of the microcomposite material and PMMA.

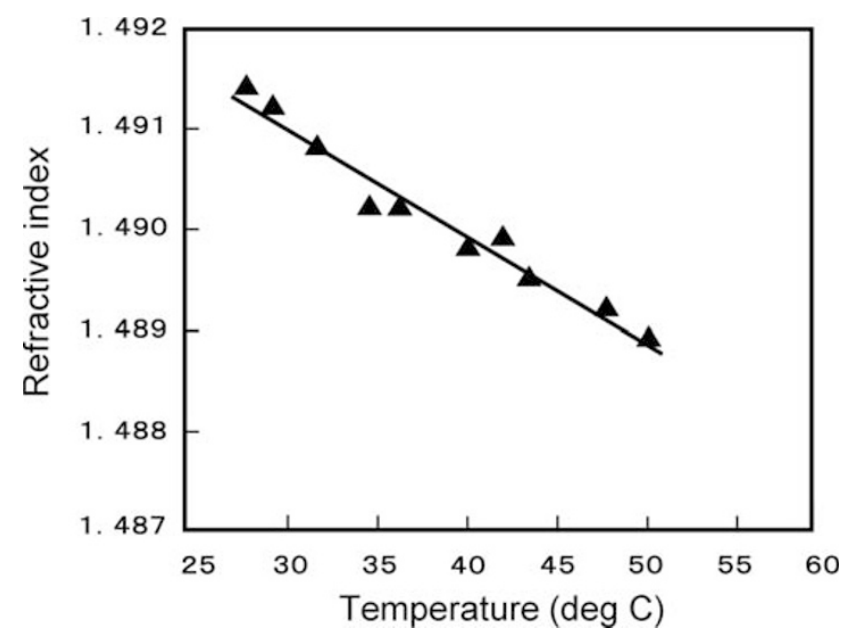

Figure 4 The dependency of refractive index variation on temperature for PMMA. The triangle symbols show the actual measurement value.

Diffusion equation. Generally, assuming that the length of the cylinder is infinite, the diffusion phenomenon in the cylinder shape was given by the diffusion equation (Equation 2), using cylindrical coordinates. In Equation 2, $C$ is the concentration of the diffusion material at position $r, D$ is the diffusion constant, $t$ is the diffusion time and $r$ is the distance from the center axis of the cylinder.

$$
\frac{\partial C}{\partial t}=D\left(\frac{\partial^{2} C}{\partial r^{2}}+\frac{1}{r} \frac{\partial C}{\partial r}\right)
$$

The simulation was set so that diffusion started at $t=0$, and M2 monomer concentration on the outside of the gel matrix was always set to $100 \%$ when $t>0$. M2 monomer was also set to diffuse at rate $P$, which is defined as the ratio between the concentration of M2 monomer in the outermost layer of the gel matrix and its surrounding region.

First, the composition distribution was obtained from the simulation program that determined $D$ and $P$. Second, the obtained

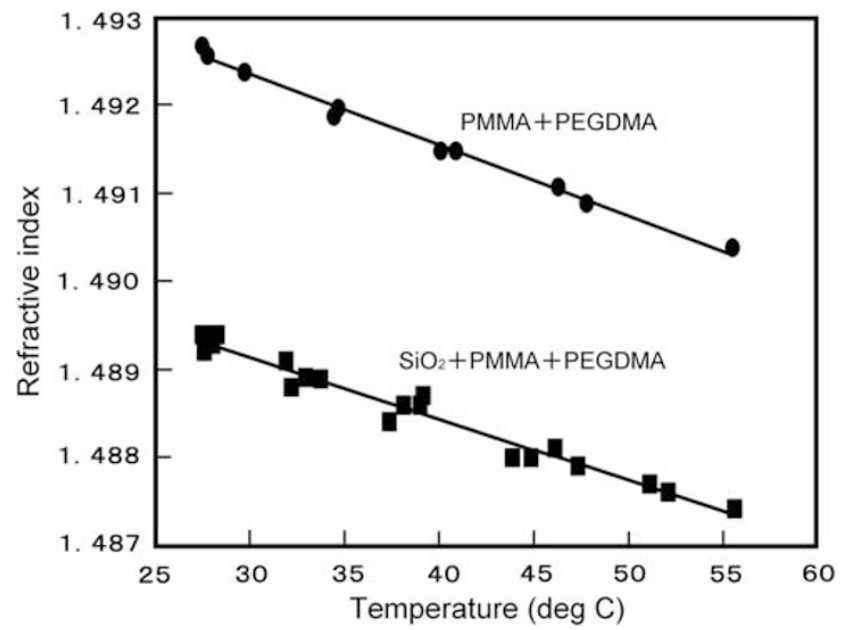

Figure 5 The dependency of refractive index variation on temperature for microcomposite material $\left(\mathrm{SiO}_{2}+\mathrm{PMMA}+\mathrm{PEGDMA}\right)$ and polymer (PMMA+ PEGDMA).

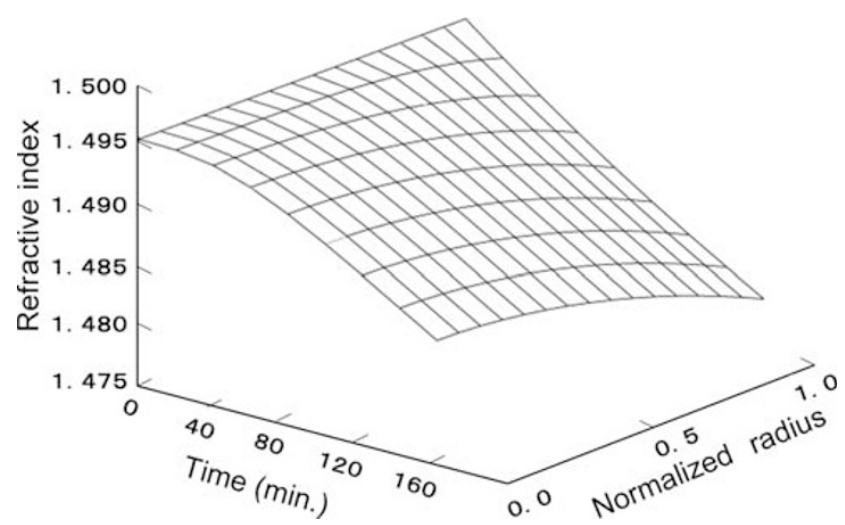

Figure 6 The refractive index profile transition during the diffusion process (calculation data).

composition distribution was converted into the refractive index profile by using the Lorentz-Lorenz equation, and $D$ and $P$ were decided by matching them to the refractive index profile actually measured. The acceleration of polymerization was confirmed about $20 \mathrm{~min}$ after the initiation of polymerization; these $20 \mathrm{~min}$ were added to the diffusion time in the simulation.

Results of the simulation. The $D$ and $P$ were determined by simulating the refractive index profiles of the microcomposite cylindrical GRIN lenses with diffusion times of both 20 and $60 \mathrm{~min}$. $D$ was found to be $2.6 \times 10^{-5} \mathrm{~cm}^{2} \mathrm{~s}^{-1}$ and $P$ was found to be $4.1 \times 10^{-4} \mathrm{~s}^{-1}$, and both values were within the vicinity of those previously reported. ${ }^{9}$ Figure 6 shows the refractive index profile of a sample with a diffusion time of $160 \mathrm{~min}$, simulated by using these values for $D$ and $P$. Here, the vertical axis indicates the refractive index, the right horizontal axis indicates the position, and the left horizontal axis indicates the diffusion time.

From the perspective of the position and the refractive index distribution, the $g$ value is large because of M2 monomer not being able to reach the central region of the cylinder during the first stage of diffusion. However, taking into consideration that the minimum 
diffusion time is $20 \mathrm{~min}$, the maximum value of $g$ was calculated to be 3.7. Simulations were further progressed to a diffusion time of $360 \mathrm{~min}$ to show that $g$ decreases up to 1.6 with an increase in diffusion time. This result indicates the possibility that, with a certain diffusion time, the most optimized $g$ value can be achieved, or a $g=2$ profile. From these results, we predicted that it was necessary to set the diffusion time to $40 \mathrm{~min}$ to obtain the best profile (substantial diffusion time $=60 \mathrm{~min}$ ).

Obtained samples. In total, three samples were fabricated, each with a different diffusion time (20, 40 and $60 \mathrm{~min})$. The dependence of the $g$

Table 2 Comparison between experimental and calculated values of the refractive index coefficient $(g)$ and refractive index difference $(d n)$ on diffusion time

\begin{tabular}{lcccccc}
\hline & \multicolumn{2}{c}{ Experimental value } & & \multicolumn{2}{c}{ Calculated value } \\
\cline { 2 - 3 } Diffusion time & $\mathrm{g}$ value & $\mathrm{dn}$ & & $\mathrm{g}$ value & $\mathrm{dn}$ \\
\hline $40 \mathrm{~min}(20 \mathrm{~min}+20 \mathrm{~min})$ & 2.35 & -0.031 & & 2.35 & -0.028 \\
$60 \mathrm{~min}(40 \mathrm{~min}+20 \mathrm{~min})$ & 2.00 & -0.031 & & 2.00 & -0.030 \\
$80 \mathrm{~min}(40 \mathrm{~min}+20 \mathrm{~min})$ & 1.85 & -0.027 & & 1.83 & -0.028 \\
\hline
\end{tabular}

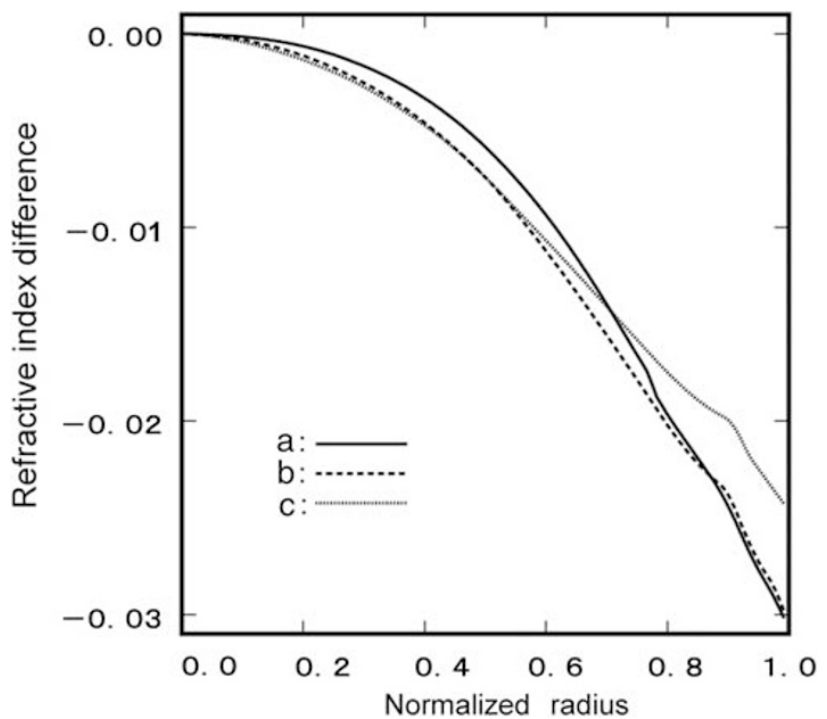

Figure 7 The refractive index profiles of three samples under the same conditions, except diffusion time (a: $20 \mathrm{~min}$; b: $40 \mathrm{~min}$; c: $60 \mathrm{~min}$ ). Source: Polymer Preprints, Japan, 56, 1194 (2007). and the refractive index difference $d n$ on diffusion time are shown in Table 2. Both $g$ and $d n$ were calculated from the obtained samples and by simulation. Figure 7 shows the refractive index profiles of the obtained GRIN rod lens. The sample with a 40-min diffusion time had a GRIN profile, and its calculated $g$ value is in good agreement with simulation results. Figure 8 shows the images transmitted through the three samples (diffused for 20, 40 and $60 \mathrm{~min}$ ). As shown in Figure 8, sample B has a clear image with hardly any distortion, whereas sample A shows a pincushion distortion and sample C shows a barrel distortion. Hence, the refractive index profile of microcomposite cylindrical GRIN lenses fabricated by this method can be controlled by diffusion time.

Moreover, these obtained samples indicate that a wide variety of refractive index profiles could be formed by combining two monomers with different characteristics. The refractive index profile is controlled by controlling the refractive index difference between the central and peripheral region of the sample. This phenomenon can be realized by balancing the refractive index of M1 and M2 monomer. Two examples of controlling the refractive index profile by monomer combination are given below.

Figure 9 shows the refractive index profile of a polymer formed using MMA as M1 and St as M2. A GRIN lens with concave lens-like features could be fabricated by this combination because St has a high refractive index, which increases the refractive index of the peripheral

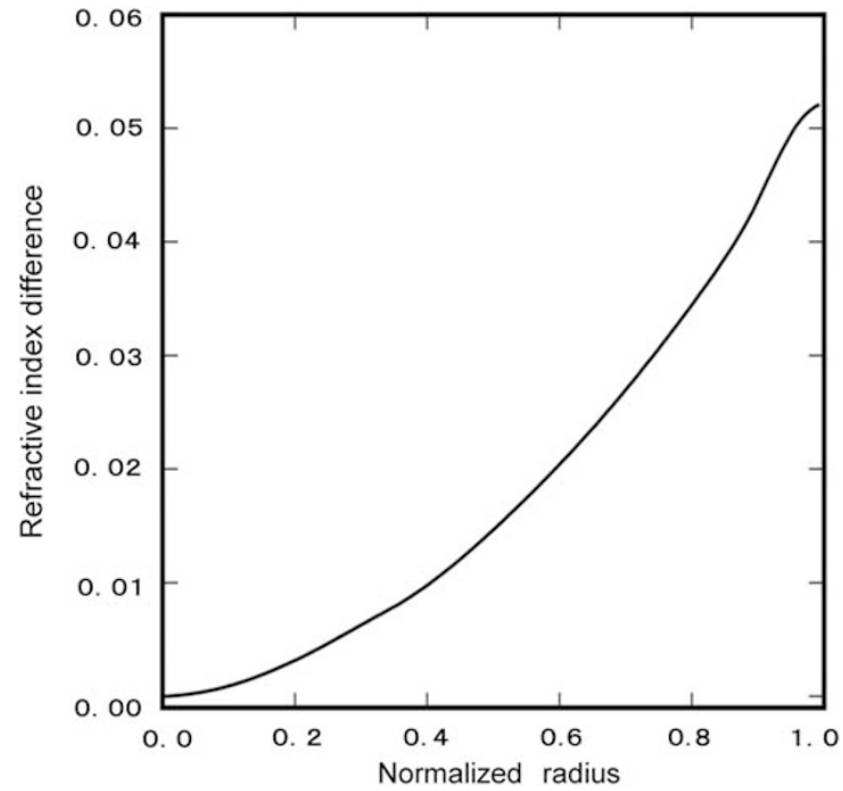

Figure 9 The refractive index profile of the $\mathrm{SiO}_{2}-\mathrm{MMA}-\mathrm{St}$ monomer system. a

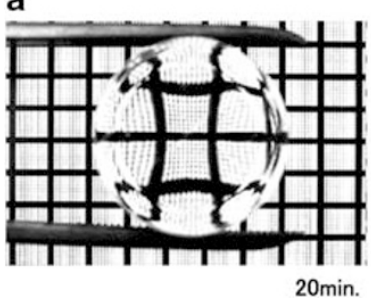

b

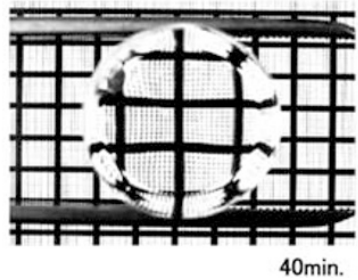

C

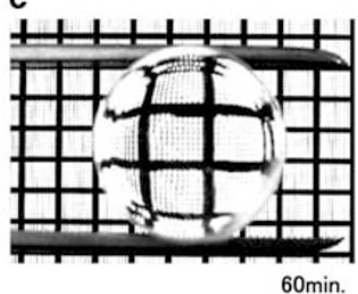

Figure 8 The grid image observed through the lenses shown in Figure 7 (a: 20 min; b: 40 min; c: 60 min). Source: Polymer Preprints, Japan, 56, 1194 (2007). 


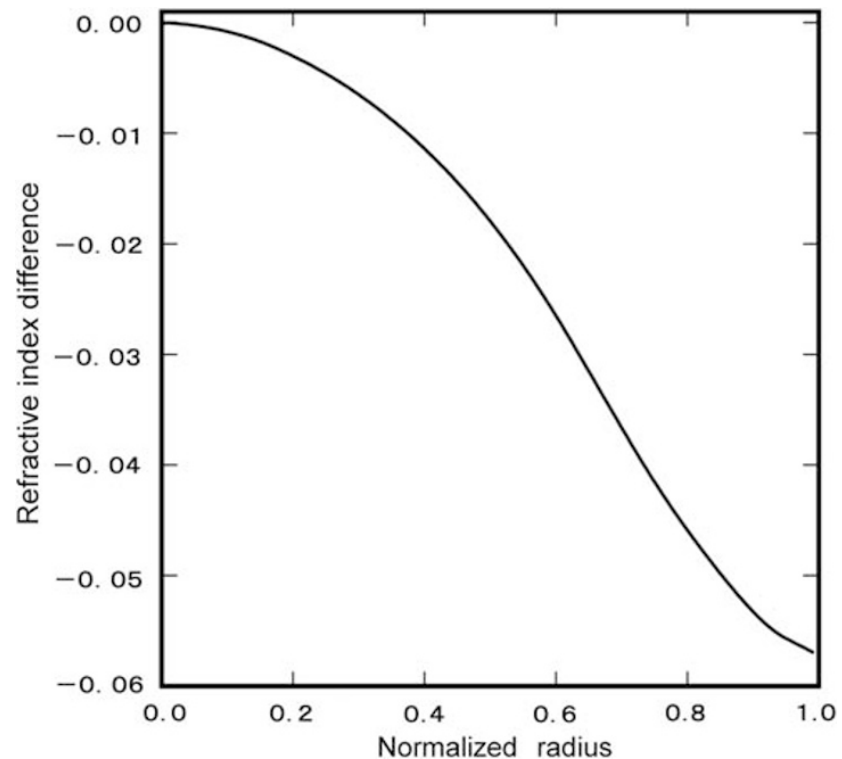

Figure 10 The refractive index profile of the $\mathrm{SiO}_{2}$-St-4FMA monomer system.

region of the sample. Figure 10 shows the refractive index profile of a polymer with St as M1 and 4FMA as M2. A GRIN lens with convex lens-like features could be fabricated by this combination because the refractive index of St is extremely high. The numerical aperture of the obtained GRIN lens was about 0.4.

\section{CONCLUSIONS}

In this study, we proposed a new fabrication method for organicinorganic microcomposite GRIN lenses. There is no specific limitation on the organic monomers that can be used by this method to obtain a refractive index profile. Hence, most organic monomers are applicable for this method. We have shown that this method can be used to successfully fabricate GRIN lenses with high transparency and high thermal stability-two essential optical characteristics-due to the formation of an organic-inorganic microcomposite structure. In addition, it is possible to control the refractive index profile coefficient, which was shown using simulations based on the diffusion equation.

With this method, we have successfully fabricated an MMA/4FMAbased microcomposite GRIN lens with the controlled refractive index profile coefficient of 2, with a refractive index difference of 0.03 . Another microcomposite GRIN lens, based on St-4FMA, was also fabricated with a refractive index difference of 0.057 . Moreover, we have shown that an MMA-St-based microcomposite GRIN lens with concave lens characteristics could be fabricated using this method. As shown clearly from these results, the GRIN lenses proposed in this paper highlight the opportunities for many practical applications of GRIN lenses.

1 Koike, Y. in Polymers for Lightwave and Integrated Optics: Technology and Applications, (ed. Hornak, L. A.), Ch. 3, 71-104 (Marcel Dekker Inc., New York, 1992).

2 Wu, S. P., Nihei, E. \& Koike, Y. The large dimensional GRIN polymer,. Polym. J. 27, 21-25 (1995).

3 Wu, S. P., Nihei, E. \& Koike, Y. Large radial graded-index polymer. Appl. Opt. 35, 28-32 (1996)

4 Peirce, J. R. Modes in sequence of lenses. Proc. Natl Acad. Sci. 47, 1808-1813 (1961).

5 Miller, S. E. Light propagation in generalized lens-like media. B. S. T. J. 40, 2017-2064 (1965).

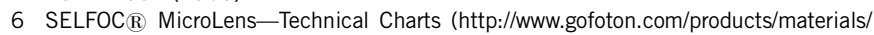
micro lenses charts.html) Accessed 6th Jun 2010.

7 Asakura, K., Nihei, E., Harasawa, H., Ikumo, A. \& Osanai, S. Nonlinear Dynamics in Polymeric Systems, (ed. Pojman, J. A., Tran-Cong-Miyata, Q.), ACS Symposium Series 869, pp. 135-146 (American Chemical Society, Washington, DC, 2003).

8 Kang, E., Bae, J. Y. \& Bae, B. Measurement of thermo-optic coefficients in sol-gel hybrid glass films. J. Sol-Gel Sci. Tech. 26, 981-984 (2003).

9 Asai, M., Hirose, R., Kondo, A. \& Koike, Y. High-bandwidth graded-index plastic optical fiber by the dopant diffusion coextrusion process. J. Lightwave Tech. 25, 3062-3067 (2007). 